\title{
Hydromechanical substantiation of the microcrack-fluid effect
}

\author{
R.S. Gurbanov*, M.A. Mammadova** \\ *Research Institute "Geotechnological Problems of Oil, Gas and Chemistry”, Azadlig av. 20, AZ 1010 Baku, Azerbaijan, \\ E-mail: ramiz.qurbanov@yahoo.com \\ **Azerbaijan State Oil Academy, Azadlig av. 20, AZ 1010 Baku, Azerbaijan, E-mail: mamedova-1944@mail.ru \\ cross $^{\text {ref }}$ http://dx.doi.org/10.5755/j01.mech.22.6.12649
}

\section{Introduction}

Numerous problems having the theoretical and experimental importance have been solved and different hydrodynamic effects have been revealed in the gas and fluid mechanics. The experimental justification of firstly detected "microcrack-fluid" effect is discussed in this work. This effect has both scientific and national-economic values so for example in the fields for processes of oil fields development, in the lubricant technologies of machines and mechanisms, in the public health for cleaning blood vessels and etc.

\section{The known hydrodynamic effects}

The numerous new effects were detected in the hydrodynamic field of science and practice, for example: the effect being characteristic of nonlinear viscous fluids and being observed as the small fountain appearance was discovered by Alan Kay in 1963.

Henri Coanda discovered effect of the fluid and gas jet during outflowing from the nozzle in 1932. On the basis of this effect one of the branches of pneumatics is constructed and a low pressure zone is created in air conditioners, in helicopter control system instead of the antitorque rotor for wing lift increasing.

The Magnus effect occurs during flow over rotating body by flow of fluid and gas, there is formed a force affecting on the body and directed perpendicular to the direction of flow and rotational motion is created around the object.

Effect Baykotta was discovered in 1920 in studies of red blood cells sedimentation. It plays an important role in the oil industry and can accelerate the slime sedimentation [1].

Weissenberg effect is characteristic of some nonNewtonian fluids such as liquid polymers' solutions. If rotating rod put into a container with the polymers solution then fluid level begins to rise up around the rod, the solution will be drawn towards the rod.

Venturi effect occurs when fluid flows through a constricted section of pipe. This effect is used in hydrojet pumps and tankers for products of petroleum and chemical industries.

Jamin effect manifests in porous medium as a result of the radii inequality of the meniscus curvature and it creates additional resistances to movement in the pore volume.

The mentioned effects are emphasized in order to show the absolute difference of well-known effects from "microcrack-fluid" effect and research methodology detected by us.

The analysis of numerous literature data shows that the anomalous properties being different from their properties in the mechanical representations are manifested during fluid motion through the channels [2-11 and etc.]. There are different assumptions about the causes of the manifestation of anomalous fluids behavior in the channels. There are various reasoned explanations about causes of the anomalous near-wall layer formation having increased viscosity and limiting yield stress [2-4 and etc.], the molecular interaction between the fluid and the solid wall [5], the capillary resistance manifestation [6] and etc.

A number of research works have been devoted to the problems of viscous fluid motion in the cracks [1-4] but the obtained results are not sufficient for explanation of the manifestation causes of the anomalous hydrodynamic behavior of fluids in the microcrack.

It is known the research works of anomalous physical and chemical properties of water but the facts of the anomalous rheological behavior of water in microcrack aren't known.

For quantitative assessment of the additional resistances in "microcrack-fluid" system it has been firstly suggested to carry out the experimental investigations in micron size crack. It will allow to have an answer to a question on anomalous behavior of the fluid in microcracks and to develop the methods of engineering evaluations on fluid filtration in the fractured porous medium and on solution of hydrodynamics problems, on lubrication in systems of machines and mechanisms as well as for various fields of industry.

It follows that the quantitative assessment of the micro-cracked effect can create more effective new approaches of mechanics of fluid and gas in different branches of industry, in oil production technology and etc.

The effects of fluid and gas mechanics revealed firstly in the microcracks will provide the new methods' development for practically total oil recovery from fields' strata of the fractured rocks.

The experimental investigations on the unit with plane and plane-radial cracks of different opening were carried out for research of the cause of the nonlinear effects manifestation during fluids motion including water in the microcrack. [7]. The causes of the anomalous properties manifestation of Newtonian fluids and increase of rheological properties of the non-Newtonian systems in the microcracks have been shown on the basis of experimental and theoretical research works. The revealed effect gets more commercial actuality. 


\section{The methodology of the experimentations}

The experimental investigations have been carried out on the unit with plane and radial cracks of different opening $h$. Plane cracks represents two plates, each one with thickness of $4 \times 10^{-2} \mathrm{~m}$, made of rustless steel of $40 \mathrm{X}$ grade. The wetted spacers with thickness of the crack opening are laying between the plates. Crack length is $0.3 \mathrm{~m}$, width $4 \times 10^{-2} \mathrm{~m}$ width with an accuracy to $5 \times 10^{-7} \mathrm{~m}$ [7]. The experiments have been carried out in one direction in the plane crack.

The fractured model construction simulating plane-radial motion of fluid in the deformable medium has been shown in Fig. 1.

Two holes were drilled in order to control the fluid motion distribution on upper plate along 3 radii located at an angle of $120^{\circ}$. It should be noted that the crack length is $84 \mathrm{~mm}$. The circle radii are equal to 34 and $57 \mathrm{~mm}$ whereon there are holes. Moreover another holes have been located near these holes at distances of $43 \mathrm{~mm}$ from the crack center. With the objective of ensuring crack undeformability the plates have been made of $40 \mathrm{X}$ steel and have surface hardness of 40-50 units according to Rockwell after heat treatment of HFC (High-Frequency Current). For crack deformation control there were used indicating gauge fixed on the upper plate of model. The inner plates' surface was processed and was grinded with accuracy corresponding to 10 category. The cracks of the defined opening have been obtained by setting of the non-wettable layerings with $5 \times 7 \mathrm{~mm}$ dimensions between the plates that are located at the points of plane-radial crack with central angles of $120^{\circ}$. The layerings' thickness was chosen in dependence on the value of the required crack opening that was measured previously with a micrometer. Moreover the determination error of the opening value equals to 0.5 microns.

Experimental investigations in plane-radial cracks were carried out in two series: the fluid movement was modeled - the first series from the center of plane-radial crack to its contour and the second series from crack contour to center. The experiments in both series were carried out at steady-state of the fluid movement.

The experiments were carried out under isothermal conditions. All ways of motion of the investigated fluid model were in thermobath. The constant temperature was maintained by ultratermostat provided with contact thermometer that was fixed directly in termobath. The crack saturation by the investigated fluid under slight pressure with simultaneous vacuuming was made for exception of Jamin effect's. It was investigated the peculiarities of the fluid motion in microcrack with different opening.

Different pressure drops on the fractured model were created after achievement of steady state mode in the experiments' process. The appropriate volumetric fluid flow rates $\mathrm{Q}$ were measured. We note that test pressure gauges with $\delta=(0.2 \div 0.35) \%$ error have been used in the experimental studies for creating different pressure drops. The mass flow rate at different pressure drops has been weighed on the laboratory electronic scale with accuracy of $0,005 \mathrm{mg}$. The dependences between shear stress $-\tau$ and velocity gradient $-\gamma$ were gotten according to the obtained data.

Water, Newtonian oil and anomalous non-Newtonian oil with $72 \%$ resin content were used as testing fluid.

Following research work [7] where it was noted that the rheological dependence generalization on the crack opening for water, kerosene, viscous and anomalous oil fall on one line. This allows to conduct research only for water and anomalous oil in the cracks and to generalize them for all Newtonian and non-Newtonian fluids.

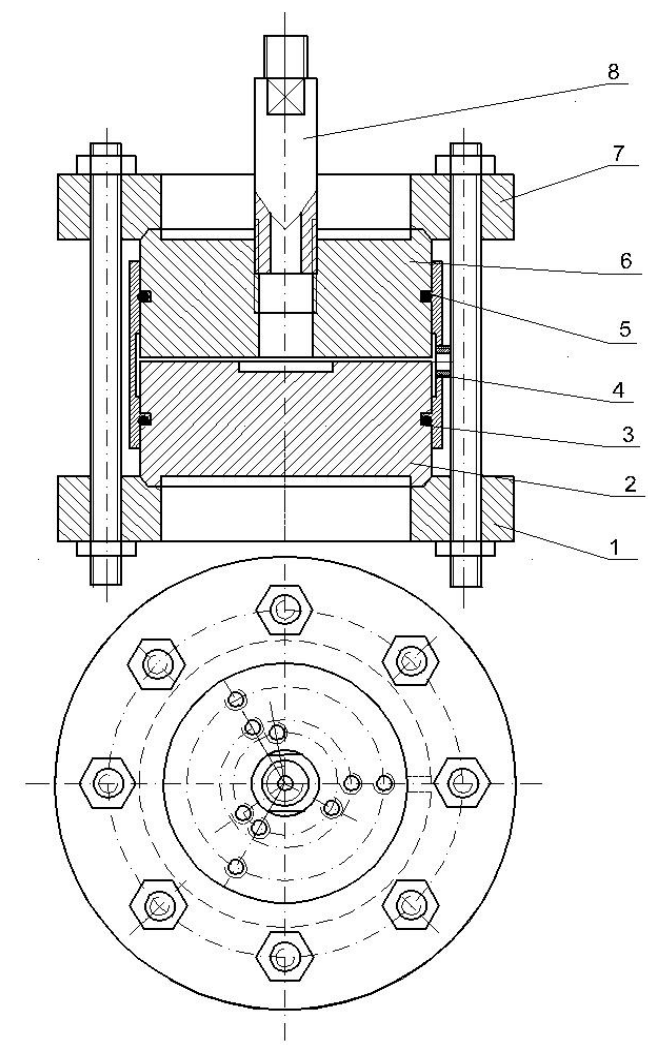

Fig. 1 Plane-radial crack model: 1, 7 - flanges; 2, 6 - plates; 3 - pressure-sealed rubber packing; 4,8 - connecting pipes; 5 - sleeve

Experimental investigations show that the flows of water and Newtonian oil in the microcrack $h<h_{c r}$ are typical for non-Newtonian systems and at large value of opening $\left(h \geq h_{c r}\right)$ for Newtonian systems.

\section{The experimental research results}

For revealing of investigated fluids in plane and plane radial cracks the results were processed in the coordinates of $\gamma-\tau$, where the average shear rate is $\gamma=6 Q / F h$ in the plane cracks and $\gamma=Q / 4 \pi r h^{2}$ in the radial; shear stress is $\tau_{w}=\Delta P h / 2 L$ in the plane cracks and $\tau_{w}=\Delta P h / L$ in the radial [12].

The experimental investigations show that the flow of water and Newtonian oil in the microcrack $\left(h<h_{c r}\right)$ are described by either power law model or by Shvedov-Bingham model and the flow in microcrack $\left(h \geq h_{c r}\right)$ is described by the parameters of fluids in bulk.

Taking into consideration the fact that the engineering oilfield problems usually occurs at high velocity gradients therefore Shvedov-Bingham model has been used in work for experimental results processing i.e. the mechanical property of the fluid in the cracks is characterized by limiting shear stress $\tau_{0 h}$ and apparent viscosity $\mu$. The limit shear stress $\tau_{0 h}$ and apparent viscosities $\mu_{h}$ at different temperatures and the crack opening have been determined on 
the basis of the $\gamma=\gamma(\tau)$ curves for Newtonian and non-Newtonian fluids.

As seen from Table, non-Newtonian properties, decreasing with the crack opening increase, are manifested during water movement in thin plane-parallel and plane-radial microcrack. The $\gamma$ dependence on $\tau$ during water motion in plane-parallel and plane-radial microcracks in the different values of the crack opening quantity at $303 \mathrm{~K}$ temperature is presented in Fig. 2 and 3. As can be seen from these figures the non-Newtonian properties, that decrease with the crack opening increase, are manifested during water movement both in rectangular and plane-radial cracks $\left(h<h_{c r}\right)$. The limiting shear stress equals to zero and the viscosity remains constant at the opening values of 22 and $25 \mu \mathrm{m}$ and higher at 313 and $306 \mathrm{~K}$ temperatures, respectively. During water movement in plane-radial microcrack at opening values of 30 and $35 \mu \mathrm{m}$ at 303 and $293 \mathrm{~K}$ temperature the limiting shear stress equals to zero and the apparent viscosity remains constant both in the first and in the second series of experiments.

The obtained dependences $\gamma=f(\tau)$ of Newtonian oil in plane-parallel microcracks at different values of crack opening at $303 \mathrm{~K}$ temperature are shown in Fig. 4. As can be seen in Fig. 4 with increasing crack opening the shear stress is decreased during Newtonian oil movement but at $130 \mu \mathrm{m}$ opening the shear stress equals to zero and the viscosity remains constant at 306 temperature.

One can be seen in Fig. 5 that in the case of nonNewtonian oil flow in plane-radial cracks with opening increasing the limiting yield stress and apparent viscosity of oil are decreased up to certain crack value. Both in the first and in the second experiments series the limiting yield stress and apparent viscosity do not depend on $h$ and remain constant at $303 \mathrm{~K}$ temperature at $180 \mu \mathrm{m}$ opening values. The limiting shear stress equals to zero and the apparent viscosity remains constant during anomalous oil flow in plane-parallel crack at 130 and $160 \mu \mathrm{m}$ opening values at 313 and $306 \mathrm{~K}$ temperatures, respectively [7]. So it has been experimentally determined that there is a critical value $\left(h_{c r}\right)$ of opening quantity below which the limiting yield stress $\tau_{0 h}$ and apparent viscosity $\mu_{h}$ are appreciably increased. It has also been established that during the Newtonian fluids motion in cracks at $h<h_{c r}$ there are manifested the non-Newtonian properties and with increase of the crack opening $h$ up to $h_{c r}$ non-Newtonian properties are decreased but and at $h>h_{c r}$ these properties are disappeared. But during the nonNewtonian fluids motion the anomalous properties are increased and with increasing crack opening they are decreased up to the initial value.

As seen from Figs. 2 and 5 the dependences obtained at injection (the curves 1-4) and at production (the curves $1^{\prime}-4^{\prime}$ ) differ appreciably from each other and the difference of $\tau_{0 h}$ and $\mu_{h}$ values is explained by initiation of local resistances and by change of fluid motion direction.

\section{The results' generalization of the determined fluids flow in plane-radial microcracks}

The experimental investigations results have been generalized during motion of water, Newtonian and anomalous oil in microcrack.

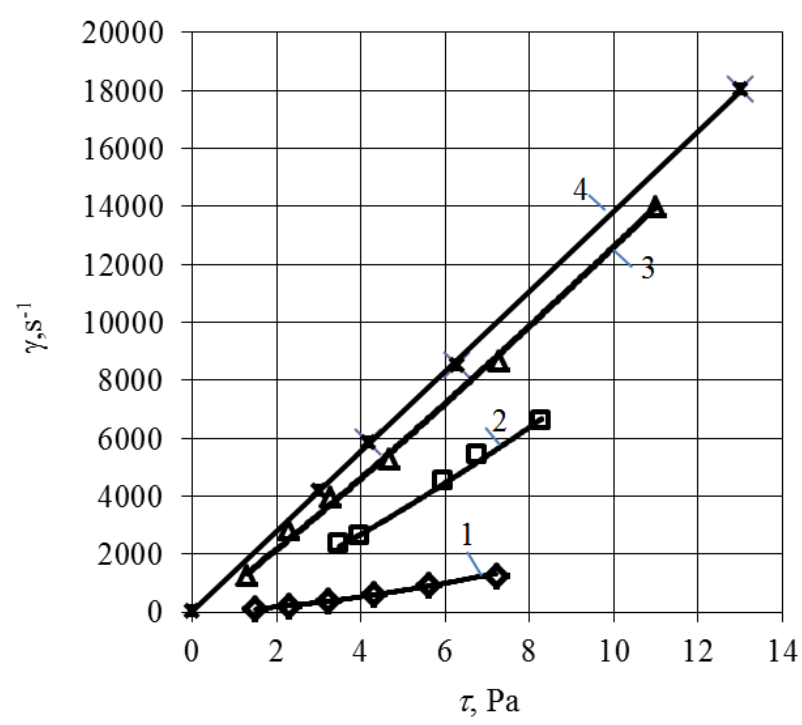

Fig. 2 The dependences of $\gamma$ from $\tau$ during water movement in plane-parallel cracks $(T=303 \mathrm{~K})$, at opening values, microns: 10 (curves 1), 15 (curves 2), 20 (curves 3 ) and 25 (curves 4$) ; h_{c r}=25 \mu \mathrm{m}$

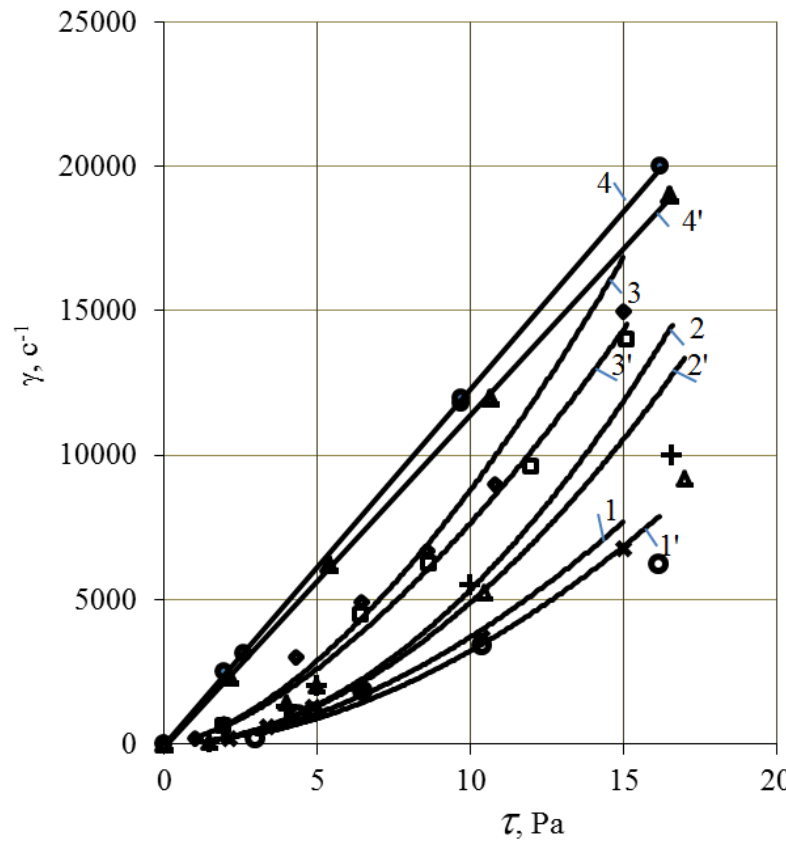

Fig. 3 The dependences of $\gamma$ from $\tau$ during water movement in plane-radial cracks $(T=303 \mathrm{~K})$, at opening values, microns: 10 (curves $1,1^{\prime}$ ), 15 (curves 2,2'), 20 (curves $3,3^{\prime}$ ) and 30 (curves $\left.4,4^{\prime}\right) ; h_{c r}=30 \mu \mathrm{m}$

There have been plotted the dependences diagrams of the dimensionless values' change $\tau_{0 h} / \tau_{0 \max }$ (for water and Newtonian oil) and $\tau_{0 h} / \tau_{0 c r}$ (for anomalous oil) as well as $\mu_{h} / \mu_{c r}$ depending on the crack opening ratio to the critical value $-h / h_{c r}$, respectively at 293 and $303 \mathrm{~K}$ temperatures. The dependences diagrams of $\tau_{0 h} / \tau_{0 \max }=f\left(h / h_{c r}\right)$, $\tau_{0 h} / \tau_{0 c r}=f\left(h / h_{c r}\right)$, and $\mu_{h} / \mu_{c r}=f\left(h / h_{c r}\right)$ consist of two straight lines, the first straight line is characteristic of the opening value with size of $0 \leq h / h_{c r}<1$, where all fluids have changed their mechanical behavior. Therefore water and all viscous fluids became anomalous and the anomalous fluids increased the non-Newtonian properties. The second straight line is characteristic of the crack opening values with $h / h_{c r} \geq 1$ sizes thereby water and all viscous and 
anomalous fluids restore their mechanical behavior i.e. the second straight line is not a continuation of the first straight line because of these lines are characterized by different behavior of the fluid.

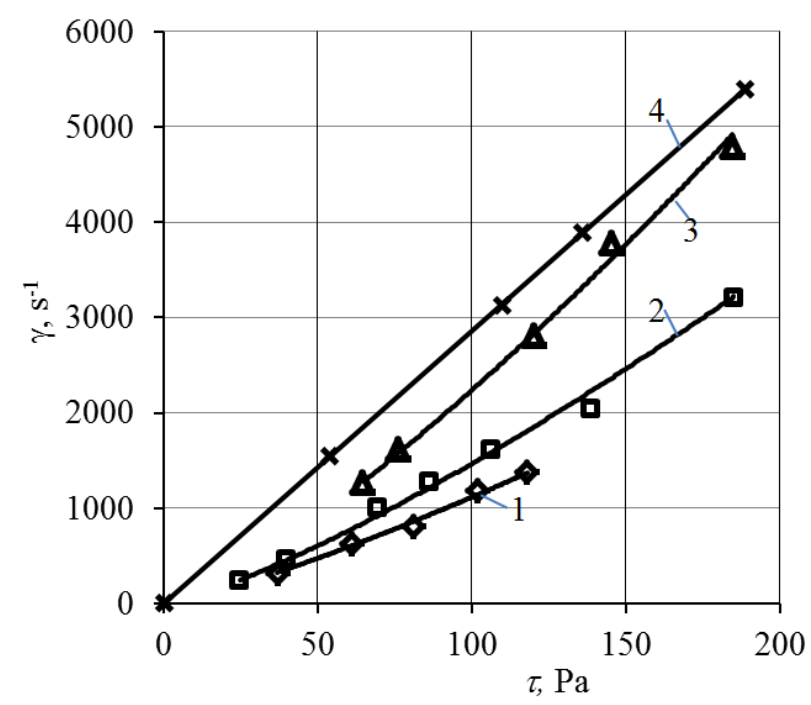

Fig. 4 The dependence of $\gamma$ from $\tau$ Newtonian oil to planeparallel microcracks $(T=303 \mathrm{~K})$ at different values of the crack opening:1, 2, 3 and 4 values of crack opening 40, 65, 90 and 130 microns, respectively. $h_{c r}=130 \mu \mathrm{m}$

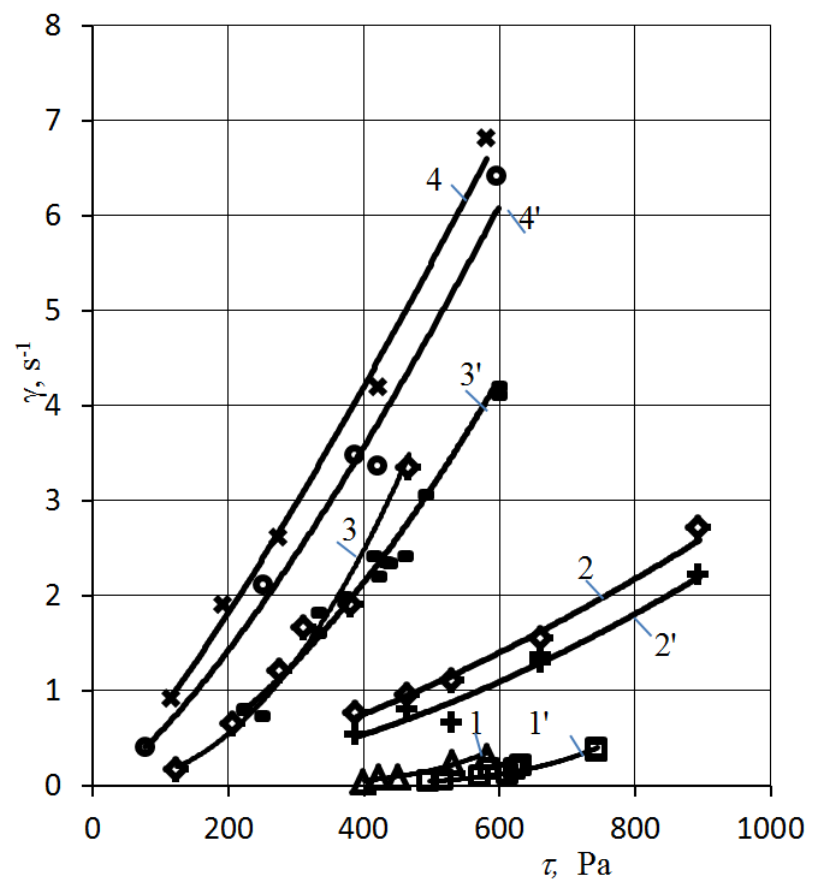

Fig. 5 The dependence of $\gamma$ from $\tau$ anomalous oil in planeradial cracks $(T=303 \mathrm{~K})$ at different values of crack opening: $1.1^{\prime} ; 2.2^{\prime} ; 3.3^{\prime} ; 4.4^{\prime}$ values of crack opening 90, 120, 160 and 180 microns, respectively. $h_{c r}=180 \mu \mathrm{m}$

The values of $a_{1}, a_{2}$ and $b_{1}, b_{2}$ coefficients during movement of Newtonian and non-Newtonian fluids in plane-radial and plane-parallel cracks

\begin{tabular}{|c|c|c|c|c|c|c|c|c|c|c|c|c|c|}
\hline \multirow{6}{*}{ 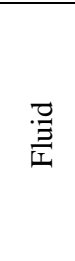 } & \multirow{6}{*}{ 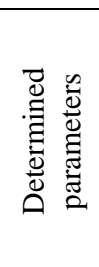 } & \multicolumn{12}{|c|}{ Coefficients } \\
\hline & & \multicolumn{8}{|c|}{ at plane-radial } & \multirow{2}{*}{\multicolumn{4}{|c|}{$\begin{array}{c}\text { at plane-parallel } \\
\text { at production }\end{array}$}} \\
\hline & & \multicolumn{4}{|c|}{ at injection } & \multicolumn{4}{|c|}{ at production } & & & & \\
\hline & & \multicolumn{12}{|c|}{ Temperatures, K } \\
\hline & & \multicolumn{2}{|c|}{293} & \multicolumn{2}{|c|}{303} & \multicolumn{2}{|c|}{293} & \multicolumn{2}{|c|}{303} & \multicolumn{2}{|c|}{306} & \multicolumn{2}{|c|}{313} \\
\hline & & $a$ & $b$ & $a$ & $b$ & $a$ & $b$ & $a$ & $b$ & $a$ & $b$ & $a$ & $b$ \\
\hline \multirow{2}{*}{$\begin{array}{l}\overline{\vec{e}} \\
\frac{\pi}{3}\end{array}$} & $\frac{\tau_{0 h}}{\tau_{0 \max }}$ & 1.212 & 1.216 & 1.132 & 1.165 & 1.184 & 1.212 & 1.319 & 1.334 & 1.104 & 1.105 & 0.909 & 0.898 \\
\hline & $\frac{\mu_{h}}{\mu_{c r}}$ & 3.067 & 2.657 & 2.462 & 1.583 & 2.821 & 2.098 & 3.479 & 2.269 & 5.060 & 4.224 & 4.744 & 4.075 \\
\hline \multirow{2}{*}{ 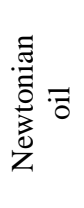 } & $\frac{\tau_{0 h}}{\tau_{0 \max }}$ & & & & & & & & & 1.02 & 0.971 & 1.01 & 0.990 \\
\hline & $\frac{\mu_{h}}{\mu_{c r}}$ & & & & & & & & & 2.18 & 1.2 & 3.18 & 2.28 \\
\hline \multirow{2}{*}{ 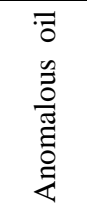 } & $\frac{\tau_{0 h}}{\tau_{0 c r}}$ & - & - & 10.017 & 9.266 & - & - & 11.028 & 10.237 & 2.570 & 1.168 & 2.470 & 1.390 \\
\hline & $\frac{\mu_{h}}{\mu_{c r}}$ & - & - & 14.460 & 14.242 & - & - & 14.198 & 14.019 & 0.010 & 1.020 & 0.420 & 0.580 \\
\hline
\end{tabular}

The following below-mentioned equations have been obtained for plane-parallel and plane-radial cracks:

For water and viscous oil:

$$
\frac{\tau_{0 h}}{\tau_{0 c r}}=a_{1}-b_{1} \frac{h}{h_{c r}} ;
$$

$$
\frac{\tau_{0 h}}{\tau_{0 \max }}=a_{1}-b_{1} \frac{h}{h_{c r}} ;
$$

For water, viscous and anomalous oil:

For anomalous oil: 


$$
\frac{\mu_{h}}{\mu_{c r}}=a_{2}-b_{2} \frac{h}{h_{c r}},
$$

where $\tau_{0 \max }$ - the largest conventional value of the limiting shear stress $\tau_{0}$ at $h=10 \mu \mathrm{m}$ crack opening, Pa. $\tau_{0 \mathrm{cr}}$ - the values of the limiting shear stress at the critical value of the crack opening, $\mathrm{Pa}$.

Based on the experiments' results there have been determined the values of the $a_{1}, a_{2}$ and $b_{1}, b_{2}$ coefficients for plane-radial and plane-parallel cracks which have been presented at Table.

It should be noted that on the basis of experimental investigations of oil samples taken from wells there are determined $\mu_{c r}$ and $h_{c r}$ values on the fracture model and there are discovered belonging of this oil to the non-Newtonian or Newtonian. In this case the boundary condition is: the critical size of the crack opening, i.e. water and viscous fluid behave as anomalous fluids during flowing in crack with the dimension of $h<h_{c r}$ and the anomalous fluids increase nonNewtonian properties. The water, viscous and non-Newtonian fluids restore their initial properties in crack with $h \geq h_{c r}$ dimension.

\section{Results discussion}

From the models of the anomalous behavior of fluids it follows that in crack with small thickness the fluid under the action of the forces applied at the ends of crack in the conditions of comparatively low average shear rates can behaves like a stable compressed rod and has certain form of equilibrium in crack. The stable rod-shaped form of fluid equilibrium depends on the crack thickness. If the thickness of thin crack is small, then applied forces at the crack ends press the fluid rod to the crack walls. The changes occur in the fluid jet properties due to changing its structure and friction forces on the walls. With increasing crack thickness, the micro-cracked effect disappears. Between these two equilibrium states there exists so-called critical wherein fluid can preserve initial properties. This crack thickness is called as the critical.

As a result of experimental investigations it has been revealed that the crack opening is one of the main indices characterizing the fluid flow properties in the fractured system. The research and assessment of the influence of opening on the fluid properties in microcrack allow to substantiate scientifically and to develop different new technological processes.

The experimental investigations were carried out with the aim of research of the micro-cracked effect influence during water movement in the plane-radial cracks with alternating opening. The fluid flows through the cracks with $h<h_{c r}=15 \mu \mathrm{m}$ opening to other crack with $h=h_{c r}=30 \mu \mathrm{m}$ opening then to the following one with $h<h_{c r}=20 \mu \mathrm{m}$. The experiments results were processed in the coordinates of $\gamma-\tau$ at $Q=$ const and it was determined that for various values of crack opening the experimental data obtained for water didn't fall on straight line just as it was in the rectangular crack [7] so in the plane-radial crack. This is due to change of fluid flow properties owing to the change of its structure and the friction forces on the crack walls. The persistence of "crack-water" effect has been shown by this experiment.

The discovered "microcrack-fluid" effect will be taken into consideration in the practice of oil production for oil recovery factor increase.

So it has been determined that the cause of the anomalous fluids behavior in the microcrack with $h<h_{c r}$ opening is effect of "microcrack-fluid of R.S. Gurbanov and M.A. Mammadova".

The results efficiency was tested in experiments for assessment of the influence methods' success on CCD.

Research results and determination method of the effective opening value corresponding to improved filtration properties and rheological parameters of oil under real conditions may be used for the projects planning of the micro-cracked fields' development.

The possibility for determination of the rheological parameters of filtration systems and crack opening under formation conditions are revealed on the basis of the developed "Methodological guideline according to the fields' development of the fractured rocks with Newtonian and nonNewtonian oils". The proposed method allows to estimate the effective value of the crack opening corresponding to the improved filtration properties upon which it can be inferred by necessity and multiplicity of carrying out of the hydraulic formation fracturing or acid treatment of CCD.

According to determined data the cracks opening value $h$ is compared with critical crack opening $h_{c r}$. The filtration characteristics of bottom-hole formation zone deteriorate significantly in the case of $h \leq h_{c r}$ and therefore measure carrying-out is considered to be appropriate. The results of timely decision making on was successful. At $h>h_{c r}$ carrying out of measure was considered as unfounded therefore it wasn't conducted.

During 2013 in "Siyazanneft" OGPD (Oil-and-Gas Production Department) the results of hydraulic fracturing of formation obtained from 100 wells were unsuccessful only in 50 wells. With the aim of cause revealing of the conducted measures ineffectiveness on the basis of the defined research data of these wells $Q=f(\Delta P)$ there were determined cracks openings. It was found that crack opening in all 50 wells was above critical value therefore it wasn't necessary to conduct these ineffective HFF (Hydraulic Fracturing of Formation) measures on the basis of the developed approach.

In 2014 it was planned carrying out of Hydraulic Fracturing of Formation fracturing in 19 wells of fields in "Siyazanneft" OGPD (Oil-and-Gas Production Department). The crack opening was determined on the basis of the developed methodical guideline. According to the research data of 13 wells it emerged that the crack opening was $h>h_{c r}$ and carrying out of measure was considered as necessary. The crack opening in other 6 wells was $h>h_{c r}$ and carrying out of measure was considered as unpractical. As a result of the proposed approach according to "Methodological guideline on the fields' development of the fractured rocks with Newtonian and non-Newtonian oils" it was early defined to carry out HFF in 13 wells that allowed to get additionally 1047.2 tons of oil and to reduce the costs planned for HFF carrying out in 6 wells.

\section{Conclusions}

1. The bases of fluids mechanics in the microcracked channels and low permeable porous media have been developed on the basis of the experimental investigations. 
2. Non-Newtonian properties are manifested during viscous fluids motion in the microcrack with $h \leq h_{c r}$ opening but non-Newtonian properties are increased for anomalous fluids and the mentioned effects are absent at $h>h_{c r}$.

3. There is critical crack opening for fluids that is 35 and $30 \mu \mathrm{m}$ at 293 and $303 \mathrm{~K}$ temperatures for water, respectively; it is 130 and $180 \mu \mathrm{m}$ for Newtonian and nonNewtonian oil at $303 \mathrm{~K}$ temperature, respectively.

4. The obtained results allow to develop the evaluation procedure of the cracks opening and permeability of wells drainage zone: for effective operability of the lubricant systems of machines and mechanisms; for cleaning thrombi from blood vessels, for successful carrying out of the different methods of the influence on the mentioned processes.

\section{Reference}

1. Hanson, P.; Trigg, T.; Rachal, G.; Zamora M. 1990. Investigation of Barite Sag in Weighted Drilling Fluids in Higlu Deviated Wells//SPE paper 20423 presented at the SPE Annual Conference and Exhibition. New Orleans, Sept.-P.23-16. https://ru.wikipedia.org/wiki2 .

2. Markhasin, I.L. 1977. Physical-Chemical Mechanics of Oil Reservoir. M.: Nedra, p. 213. (in Russian).

3. Romm, E.S. 1966. Filtration Properties of the Fractured Rocks. M.: Nedra, p. 284. (in Russian).

4. Loytsyanskiy, L.G. 2003. Fluid and Gas Mechanics. P.840- M.: Drofa. (in Russian).

5. Gimatudinov, Sh.K. 1963. Physics of the Oil Reservoir. M.: Gostoptekhizdat, p. 274. (in Russian).

6. Svalov, A.M. 2011. The capillary effects in the fractured rocks, Oil Industry 1: 59-63. (in Russian).

7. Mammadova, M.A. 2013. About anomalous fluids' behavior in the microcracks, Oil-field Engineering 2: 3842. (in Russian).

8. Kashirina, K.O.; Telkov, A.P. 2010. A brief analysis of some analytical solutions of the problems about fluid flow to vertical crack of the hydraulic fracturing in the productive stratum, Oil-field Engineering 9:9-13. (in Russian).
Zaslavskiy, M.Yu.; Tomin, P.Yu. 2010. About modeling of multiphase filtration processes in the fractured media in relation to problems of deposit's model adaptation, Preprints of Keldysh Institute of Applied Mathematics 45: 20. (in Russian).

9. Teodorovich, E.V.; Trofimov, A.A.; Shumilin I.D. 2011. The flat crack form of hydraulic fracturing in the elastic impermeable medium at different injection rates, Mechanics of Fluid and Gas 4: 109-113. (in Russian).

10. Rustamov, I.F.; Vladimirov, I.V.; Egorov, A.F., Magzyanov, I.A. 2013. About cracks system role in formation of oil flow from the low-resistivity reservoirs, Oil-field Engineering 3: 91-95. (in Russian).

11. Mirzajanzade, A.Kh.; Gevinyan, G.M. et al. 1977. Hydraulics of Clay and Cement Solutions, Moscow: Nedra, p. 229 (in Russian).

\section{R.S. Gurbanov, M.A. Mammadova \\ HYDROMECHANICAL SUBSTANTIATION OF THE MICROCRACK-FLUID EFFECT}

Sum mary

Firstly it has been experimentally revealed that the cause of the anomalous properties manifestation of viscous fluids and particularly water and these properties increase for anomalous fluids in the cracks is a new micro-cracked effect "fluid-medium" system. It is recommended scientific and practical results of the micro-cracked effect in "fluidmedium" system which can be considered for analysis of the different industry fields.

Keywords: microcrack opening, anomalous water, Jamin effect, limiting yield stress, apparent viscosity.

Received July 06, 2015

Accepted November 25, 2016 